\title{
SELECTIVE PHOTODISSOCIATION OF CONDENSED AND ADSORBED SMALL MOLECULES BY SPECIFIC CORE-TO-BOUND EXCITATIONS: EVIDENCE FOR ULTRAFAST DISSOCIATION CHANNELS
}

\author{
DIETRICH MENZEL \\ Physik-Department E20, Techn. Universität München, D 8046 Garching $b$. \\ München, Fed. Rep. Germany
}

\begin{abstract}
The modification of photodissociation mechanisms of small molecules by adsorption on a solid or by condensation is briefly reviewed. The coupling to the surroundings leads to quenching of easily delocalizable primary excitations so that only strongly localized channels show up in dissociation (detected by photodesorption, i.e. by the appearance of fragments in the gas phase). In reverse, fast direct dissociation channels will be less in need of such localization and will be preferred over slow channels. Investigation of dissociation induced by core excitations or core shake-ups introduces an internal time mark - core life time- - to which reaction time can be compared. The example of small molecules, such as $\mathrm{CO}$ or NO, adsorbed on transition metal surfaces is surveyed, in which complex multiple excitations predominate in dissociation. Recent results on the core-induced dissociation of condensed hydrogenic molecules such as water, ammonia, and benzene have shown the existence of preferential dissociation channels in the core-to-bound region. It is argued that these dissociation processes are at least competitive with core decay; they are termed ultrafast. It appears that they should also exist in the free molecules.
\end{abstract}

KEY WORDS: Excitations, photodissociation, condensed, adsorbed.

Photodissociation of molecules is modified by adsorption or condensation, since the coupling to the surroundings tends to quench slow dissociation channels which become active only after energy redistribution in the molecule, and emphasizes fast, direct channels. This has been demonstrated earlier by the investigation of small molecules such as $\mathrm{CO}$ and NO, adsorbed on transition metal surfaces. ${ }^{1,2}$ In these cases the main results can be summarized as follows.

1) Generally the dissociation probabilities are much smaller than in the gas phase (by factors up to $10^{3}$ or more).

2) Core excitation is more effective than valence excitation, but even in the core region the fragment ion yields do not follow the excitation cross sections, but core shake-up excitations are much more dissociative than normal core excitations (see Figure 1). 


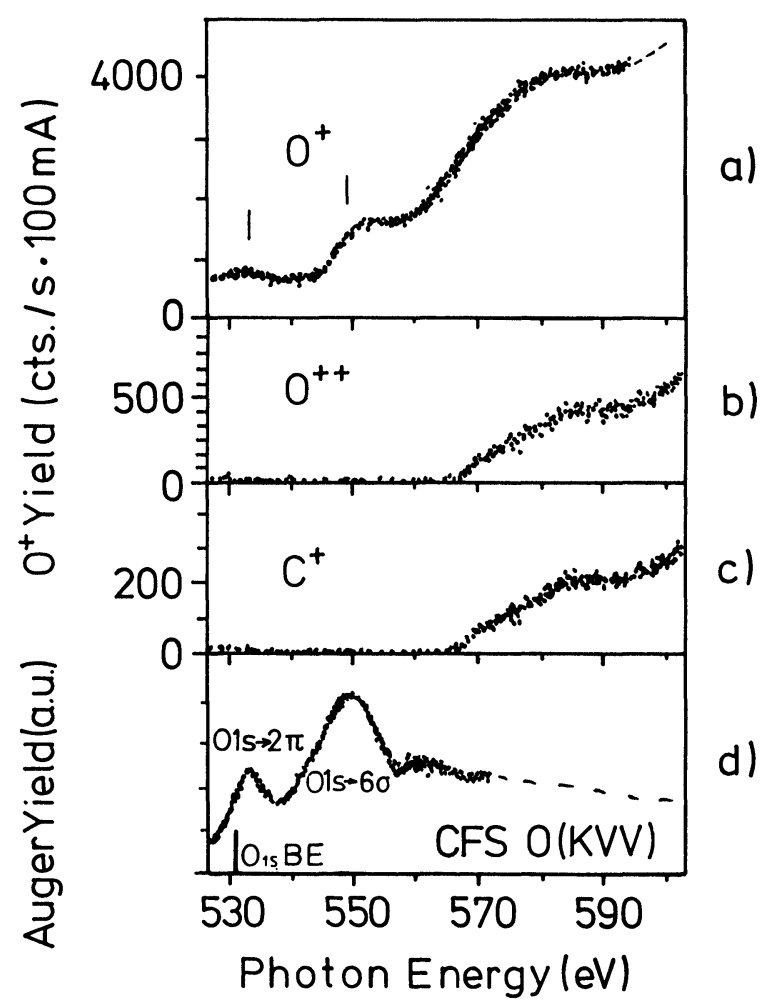

Figure 1 Yields of fragment ions from $\mathrm{CO}$ adsorbed on $\mathrm{Ru}(001)$ in the $\mathrm{O} 1 \mathrm{~s}$ range, compared to the photoabsorption as indicated by the yield of O KLL Auger electrons (bottom curve). The strong enhancement of multiple excitations above $550 \mathrm{eV}$ in the fragment yields is obvious. (From Ref. 1, with permission).

3) While the characteristic structures provided by these shake-up resonances are independent of the strength of the surface bond, the overall yield is the lower the weaker this bond; more generally:

4) Any effect which increases the coupling (increased adsorption energy; increased coverage; two-dimensional order; coadsorption of alkali atoms) decreases the dissociation yield; any effect which decreases the coupling (surface disorder or defects; vanishing lateral interactions because of low coverage; inert spacer layers) increases it.

Most of these results can be understood by the delocalization/recapture mechanism. ${ }^{3}$ It assumes that the overall dissociation probability is the product of the primary excitation probability and a factor defining the survival of the excitation at its primary location, up to the actual bond breaking event. This has two consequences: the dissociation yield per primary excitation will be increased for strongly localized excitations on the one hand, and by excitations leading to fast dissociation on the other. 
In the past the first aspect has been mainly emphasized. It makes the enhanced dissociativeness of core shake-ups, i.e. of strongly correlated multiple excitations understandable, since these themselves, as well as their core decay products, are highly localized, ${ }^{4}$ even for systems interacting strongly with continua such as adsorbates on metal surfaces. If these excitations are protected from delocalization into the metal by their correlation, then core decay to multiply charged systems can proceed which then Coulomb-explode.

As discussed in detail before, ${ }^{1,2}$ arguments can be given that this is not the complete story, but that some or all of the observed relative enhancement of dissociation must be understood by "ultrafast" events, i.e. dissociation on a time scale fast compared to or at least competitive with core decay. These arguments can be further strengthened by the study of core decay electron spectra. ${ }^{5}$ Indeed the dissociative effect of multiple excitations can also be understood by assuming that these tend to possess more strongly repulsive potential energy curves which can lead to direct dissociation; then dissociation is so fast that there is no need for increased localization. The use of core excitations in this connection is very helpful not only to pump high energies into the system, but also because the core life time introduces an additional time mark to which reaction times can be compared. We have termed reactions which are faster than core life times "ultrafast."

Interestingly, investigation of photodissociation of adsorbed and condensed hydrogenic molecules (water; ${ }^{6,7}$ ammonia $;{ }^{7}$ benzene ${ }^{8}$ ) has shown that very dissociative excitations can be found for these in the region of discrete core excitations, i.e. in the core-to-bound region below the photoionization threshold. As examples, Figure 2 shows the comparison of $\mathrm{H}^{+}$yield to Auger electron yield (representing the absorption curve) of $\mathrm{H}_{2} \mathrm{O}$ and $\mathrm{NH}_{3}$ ices around the $\mathrm{O}$ 1s and $\mathrm{N}$ 1s thresholds, respectively. The strong enhancement of the lowest core-to-bound resonance (the $1 \mathrm{~s}^{-1} 4 \mathrm{a}_{1}{ }^{+1}$ ) in both cases is clearly visible (for a discussion of the broadening and shift of the absorption curves by condensation, see Ref. 7). This excitation, or its MO, non-Rydberg part, ${ }^{6,7}$ is strongly antibonding for the $\mathrm{O}-\mathrm{H}$ or $\mathrm{N}-\mathrm{H}$ bond. In the case of benzene ${ }^{8}$ a (somewhat smaller) enhancement is found not for the lowest excitation (which in this case has $\pi^{*}$ character and has no effect on the $\mathrm{C}-\mathrm{H}$ bond) but for an excitation about $2 \mathrm{eV}$ higher which can be interpreted as the lowest $\sigma^{*}$ excitation with respect to the $\mathrm{C}-\mathrm{H}$ system. So the qualitatitive picture is clear that strong enhancement of dissociation occurs for the most strongly antibonding resonances. It should be mentioned that no such enhancement has been found for condensed $\mathrm{CH}_{4}{ }^{7}$

Based on additional findings, mainly about the dependences of ion signal on the angles of polarization and emission which lead to the conclusion of symmetry breaking for $\mathrm{H}_{2} \mathrm{O}$, we have argued ${ }^{6,7}$ that these enhancement effects can best be understood if it is assumed that dissociation starts during core life times (which are of the order of $5 \times 10^{-15} \mathrm{~s}$ ) along the strongly repulsive antibonding curve. This becomes particularly important for the light hydrogen atom. Contrary to the earlier findings of Nenner et al. ${ }^{10}$ for $\mathrm{HBr}$ and $\mathrm{CH}_{3} \mathrm{Br}$ core-induced photodissociation, however, the dissociation cannot be complete when core decay happens; otherwise the resulting ionization of the expelled hydrogen would not be understandable which must be caused by interatomic Auger decay of the separating system. This means 

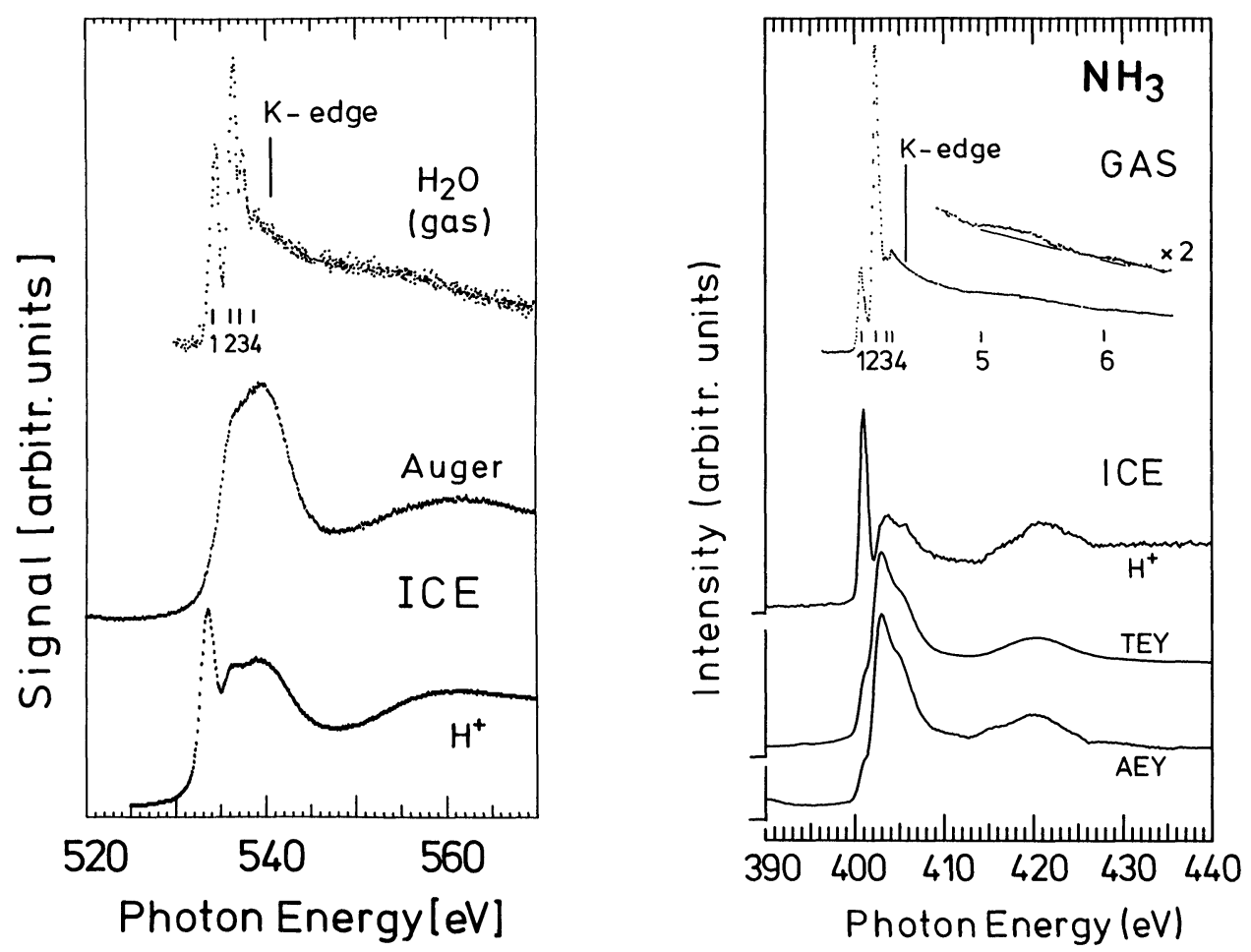

Figure 2 Photodissociation of (a) condensed water by O1s excitation; and (b) condensed ammonia by N1s excitation. The photoabsorption of the respective ice (measured by the yield of Auger electrons, center curve in (a); for $\mathrm{NH}_{3}$ also the total electron yield, TEY, is given which is more weighted towards the bulk while the AEY more towards the surface layer) is a broadened and shifted replica of the absorption of the free molecule (measured by electron energy loss spectroscopy; ${ }^{9}$ top curves). The $\mathrm{H}^{+}$yield curves of the ices (bottom curves in (a) and (b)) show pronounced threshold peaks which are assigned to the strongly dissociative $1 \mathrm{~s} \rightarrow 4 \mathrm{a}_{1}$ excitation (see text) and which correspond to an enhancement by at least a factor 10 relative to the absorption curve (From Refs. 6 and 7, with permission).

that the sequence from primary excitation via electronic decay to dissociation constitutes one coupled process; the mentioned symmetry breaking thus becomes also understandable.

It is not clear at the moment whether coupling (to the metal surface for the adsorbates; to the other molecules in the condensates) is important for this mechanism to work, or whether it only serves to make these channels more obvious, by quenching of more normal channels. Detailed corresponding photodissociation measurements on the free molecules in the gas phase are necessary to decide this question; such measurements are planned in collaborations.

In conclusion, we have shown that work on photodissociation of adsorbed and condensed molecules in the core excitation region emphasizes the contributions of fast, direct channels besides of those strongly localized by correlation, and have given evidence for ultrafast dissociation channels, i.e. events competitive with or faster than core decay. These channels should also be present in the isolated molecules. 


\section{Acknowledgements}

The important contributions of many coworkers, in particular of D. Coulman, P. Feulner, G. Rocker, R. Treichler and W. Wurth, are gratefully acknowledged. This work has been supported by the Deutsche Forschungsgemeinschaft (through SFBs 128 and 338), by the German Federal Ministry of Research and Technology (under $05466 \mathrm{CAB}$ ), and by the European Community (CEC Stimulation Project).

\section{References}

1. R. Treichler, W. Riedl, W. Wurth, P. Feulner and D. Menzel, Phys. Rev. Lett. 54, 462 (1985); R. Treicher, W. Wurth, W. Riedl, P. Feulner and D. Menzel, Chem. Physics 153, 259 (1991).

2. R. Treichler, W. Riedl, P. Feulner and D. Menzel, Surface Sci. 243, 239 (1991).

3. D. Menzel and R. Gomer, J. Chem. Phys. 41, 3311 (1964); D. Menzel, in: Desorption Induced by Electronic Transitions, DIET-I, Eds. N.H. Tolk et al.(Springer, Berlin 1983), p. 68; and in DIET-III, eds. R.H. Stulen and M.L. Knotek (Springer, Berlin 1987), p. 2; D. Menzel, Nucl. Instrum. Methods Phys. Res. B 13, 507 (1986).

4. M. Cini, Solid State Commun. 24, 681 (1977); G. A. Sawatzki, Phys. Rev. Lett. 39, 504 (1977).

5. D. Menzel, P. Feulner, R. Treichler, E. Umbach and W. Wurth, Physica Script. T 17, 166 (1987); W. Wurth, C. Schneider, R. Treichler, D. Menzel and E. Umbach, Phys. Rev. B 37, 8725 (1988).

6. D. Coulman, A. Puschmann, W. Wurth, H.-P. Steinrück and D. Menzel, Chem. Phys. Lett. 148, 371 (1988); D. Coulman, A. Puschmann, U. Höfer, H.-P. Steinruck, W. Wurth, P. Feulner and D. Menzel, J. Chem. Phys. 93, 58 (1990).

7. D. Menzel, G. Rocker, D. Coulman, P. Feulner and W. Wurth, Physica Script. 41, 588 (1990); G. Rocker, D. Coulman, P. Feulner, R. Scheuerer, Zhu Lin, and D. Menzel, in: Desorption Induced by Electronic Transitions, DIET-IV, Eds. G. Betz and P. Varga (Springer, Berlin 1990), p. 261.

8. D. Menzel, G. Rocker, H.-P. Steinrück, D. Coulman, P. Heimann, W. Huber, and D. R. Lloyd, To appear in Phys. Rev. B.

9. G. R. Wright and C. E. Brion, J. Electron Spectrsoc. Rel. Subj. 4, 25 (1974).

10. P. Morin and I. Nenner, Phys. Rev. Lett. 56, 1913 (1986); I. Nenner, P. Morin, M. Simon, P. Lablanqie, and G. G.B. deSouza, in: DIET-III, Eds. R. H. Stulen and M. L. Knotek (Springer, Berlin 1988) p. 10; and references therein. 